\title{
Clinical and metabolic features of the randomised controlled Diabetes Remission Clinical Trial (DiRECT) cohort
}

\author{
Roy Taylor ${ }^{1} \cdot$ Wilma S. Leslie ${ }^{2}$ Alison C Barnes ${ }^{3} \cdot$ Naomi Brosnahan ${ }^{2,4}$. \\ George Thom ${ }^{2} \cdot$ Louise McCombie $^{2} \cdot$ Naveed Sattar $^{5} \cdot$ Paul Welsh $^{5} \cdot$ Carl Peters $^{1}$ • \\ Sviatlana Zhyzhneuskaya ${ }^{1} \cdot$ Kieren G. Hollingsworth $^{1} \cdot$ Ahmad Al-Mrabeh $^{1}$ • \\ Angela M. Rodrigues ${ }^{6} \cdot$ Lucia Rehackova $^{6}$ • Ashley J. Adamson ${ }^{3} \cdot$ Falko F. Sniehotta $^{6}$. $^{\circ}$ \\ John C. Mathers ${ }^{7} \cdot$ Hazel M. Ross ${ }^{4} \cdot$ Yvonne McIlvenna $^{8} \cdot$ Sharon Kean $^{9} \cdot$ Ian Ford ${ }^{9}$ • \\ Alex McConnachie' ${ }^{9}$ Michael E. J. Lean ${ }^{2}$
}

Received: 25 September 2017 / Accepted: 25 October 2017 /Published online: 30 November 2017

(C) The Author(s) 2017. This article is an open access publication

\begin{abstract}
Aims/hypothesis Substantial weight loss in type 2 diabetes can achieve a return to non-diabetic biochemical status, without the need for medication. The Diabetes Remission Clinical Trial (DiRECT), a cluster-randomised controlled trial, is testing a structured intervention designed to achieve and sustain this over 2 years in a primary care setting to determine practicability for routine clinical practice. This paper reports the characteristics of the baseline cohort.

Methods People with type 2 diabetes for $<6$ years with a BMI of $27-45 \mathrm{~kg} / \mathrm{m}^{2}$ were recruited in 49 UK primary care practices, randomised to either best-practice diabetes care alone or with an additional evidence-based weight management programme (Counterweight-Plus). The co-primary outcomes, at 12 months, are weight loss $\geq 15 \mathrm{~kg}$ and diabetes remission $\left(\mathrm{HbA}_{1 \mathrm{c}}<48 \mathrm{mmol} / \mathrm{mol}\right.$ [6.5\%]) without glucose-lowering
\end{abstract}

Roy Taylor and Michael E. J. Lean are joint principal investigators and senior authors for this work.

Michael E. J. Lean

mike.lean@glasgow.ac.uk

1 Newcastle Magnetic Resonance Centre, Institute of Cellular Medicine, Campus for Ageing and Vitality, Newcastle University, Newcastle upon Tyne, UK

2 Human Nutrition, School of Medicine, Dentistry and Nursing - GRI Campus, College of Medical, Veterinary and Life Sciences, University of Glasgow, 2nd Floor, New Lister Building, Glasgow Royal Infirmary, 10-16 Alexandra Parade, Glasgow G31 2ER, UK

3 Human Nutrition Research Centre, Institute of Health and Society, Newcastle University, Newcastle upon Tyne, UK

4 Counterweight Ltd, Corby, Northants, UK therapy for at least 2 months. Outcome assessors are blinded to group assignment.

Results Of 1510 people invited, 423 (28\%) accepted; of whom, $306(72 \%)$ were eligible at screening and gave informed consent. Seven participants were later found to have been randomised in error and one withdrew consent, leaving 298 (176 men, 122 women) who will form the intention to treat (ITT) population for analysis. Mean (SD) age was 54.4 (7.6) years, duration of diabetes 3.0 (1.7) years, BMI $34.6(4.4) \mathrm{kg} / \mathrm{m}^{2}$ for all participants (34.2 (4.2) kg/m in men and $35.3(4.6) \mathrm{kg} / \mathrm{m}^{2}$ in women) and baseline $\mathrm{HbA}_{1 \mathrm{c}}$ (on treatment) $59.3(12.7) \mathrm{mmol} / \mathrm{mol}(7.6 \%$ $[1.2 \%])$. The recruitment rate in the intervention and control groups, and comparisons between the subgroups recruited in Scotland and England, showed few differences.

Conclusions/interpretation DiRECT has recruited a cohort of people with type 2 diabetes with characteristics similar to
Institute of Cardiovascular and Medical Science, University of Glasgow, Glasgow, UK

6 Institute of Health and Society, Faculty of Medical Sciences, Newcastle University, Newcastle upon Tyne, UK

7 Human Nutrition Research Centre, Institute of Cellular Medicine, Campus for Ageing and Vitality, Newcastle University, Newcastle upon Tyne, UK

8 General Practice and Primary Care, Institute of Health and Wellbeing, University of Glasgow, Glasgow, UK

9 Robertson Centre for Biostatistics, Institute of Health and Wellbeing, University of Glasgow, Glasgow, UK 
those seen in routine practice, indicating potential widespread applicability. Over $25 \%$ of the eligible population wished to participate in the study, including a high proportion of men, in line with the prevalence distribution of type 2 diabetes.

Trial registration www.controlled-trials.com/ISRCTN 03267836; date of registration 20 December 2013

Keywords Formula diet · Remission · Type 2 diabetes . Weight management

$\begin{array}{ll}\text { Abbreviations } \\ \text { ALT } & \text { Alanine aminotransferase } \\ \text { AST } & \text { Aspartate aminotransferase } \\ \text { CVD } & \text { Cardiovascular disease } \\ \text { DiRECT } & \text { Diabetes Remission Clinical Trial } \\ \text { GGT } & \gamma \text {-Glutamyl transferase } \\ \text { GP } & \text { General practitioner } \\ \text { ITT } & \text { Intention to treat } \\ \text { NECS } & \text { North East Commissioning Support } \\ \text { PCRN } & \text { Primary Care Research Network }\end{array}$

\section{Introduction}

In the early years after diagnosis type 2 diabetes has been shown to be a reversible metabolic condition $[1,2]$. Within 10 years of type 2 diabetes diagnosis, about two-thirds of individuals can return to non-diabetic levels of blood glucose control after diet-induced weight loss averaging $15 \mathrm{~kg}$ [3]. With stable reduced weight, non-diabetic blood glucose control and changes in the underlying pathophysiological abnormalities persist without glucose-lowering medications for at least 6 months [3]. However, these results have only been observed in a small number of motivated volunteers attending a specialist research unit. The practical implications for routine clinical practice of such diet-induced type 2 diabetes reversal urgently need to be established given widening public interest in this phenomenon.

Bariatric surgery of any kind can reverse metabolic abnormalities in about $70 \%$ of people with type 2 diabetes [4], with the likelihood of benefit determined primarily by the extent of weight loss and duration of diabetes [5, 6]. However, with conventional dietary advice mean weight loss is only $3-5 \%$ and remission is rare [7]. In the LookAHEAD study, a greater mean weight loss of $8 \%$ at 12 months produced remission of type 2 diabetes in $11.5 \%$ of participants [8]. The present study was designed to use a more intensive initial 'total diet replacement' approach, using a low-energy formula diet with a structured behaviour change programme for long-term weight loss maintenance, delivered in a realistic primary care setting. In a feasibility study, this programme resulted in a mean weight loss of about $17 \mathrm{~kg}$ by 12 weeks, with $40 \%$ remaining
$>10 \mathrm{~kg}$ and $33 \%>15 \mathrm{~kg}$ below baseline weight at 12 months [9]. Similar figures have been reported for a roll-out of the Counterweight-Plus programme to 288 individuals in routine primary care (L. McCombie, N. Brosnahan, H. Ross, A. BellHiggs, L. Govan, M. E. J. Lean, unpublished results). The Diabetes Remission Clinical Trial (www.controlled-trials. com/ISRCTN03267836) was designed to answer a series of linked questions [10]: What proportion of the whole population with type 2 diabetes would agree to undertake substantial weight loss? How many of these could achieve $\geq 15 \mathrm{~kg}$ weight loss and return to non-diabetic blood glucose control off medication (the twin primary endpoints) at 1 year? What happens to the major pathophysiological factors underlying type 2 diabetes during continued follow up? What are the psychological consequences of the programme and can outcomes be predicted by psychometric variables? What proportion of the intervention group remains non-diabetic after 2 years? This paper describes the recruitment process, outcomes of recruitment and baseline characteristics of the study participants.

\section{Methods}

A cluster-randomised controlled design was employed, with general practices the unit of randomisation. General practices representing populations with wide ranges of social and geographical features across Scotland and in the Tyneside region of northeast England were invited to participate by the Primary Care Research Network (PCRN) in Scotland and North East Commissioning Support (NECS) in England. Practices known to be research-active were approached first as the demands of research work by staff would be too great for many practices.

The research team met with interested practices to discuss participation details. Following these meetings, willing practices were then randomised to intervention or control. Practices randomised to the control group continued to deliver usual diabetes and weight management as per current clinical guidelines. Practices randomised to the intervention group continued to deliver usual guideline-based care in addition to an evidence-based weight management programme (Counterweight-Plus). Randomisation was conducted independently by the Robertson Centre for Biostatistics, University of Glasgow, using a minimisation method to maintain balance across groups between the two study regions (Scotland and Tyneside), including a balance of practice list sizes $(n>5700$ or $n \leq 5700)$.

Within each general practice, all people with type 2 diabetes meeting inclusion and exclusion criteria were identified by a computerised search of general practitioner (GP) records, undertaken by PCRN in Scotland and GP practice staff in Tyneside. Lists generated by the search were reviewed by GPs and any individuals recognised to be unsuitable to 
approach because of comorbidity or other practical obstacles to participation were removed.

Inclusion criteria were age 20-65 years, type 2 diabetes of duration $0-6$ years (diagnosis based on two recorded diagnostic tests using blood glucose and/or $\mathrm{HbA}_{1 \mathrm{c}}\left[\mathrm{HbA}_{1 \mathrm{c}}>48 \mathrm{mmol} / \mathrm{mol}\right.$ $(6.5 \%)$ on diet alone or $\mathrm{HbA}_{1 \mathrm{c}}>43 \mathrm{mmol} / \mathrm{mol}(6.1 \%)$ on treatment with oral glucose-lowering agents]), and a BMI of $27-45 \mathrm{~kg} / \mathrm{m}^{2}$. Potentially eligible individuals were mailed an invitation to participate with an information sheet and asked to respond using prepaid reply envelopes. Full details of methodology have been previously described [10]. A subsequent protocol amendment included the provision of a financial incentive (50 GBP Amazon retail voucher) for those recruited into the control arm. The intervention arm already provided a desirable incentive 'in kind' for participation (supply of a formula diet and potential substantial weight loss) but

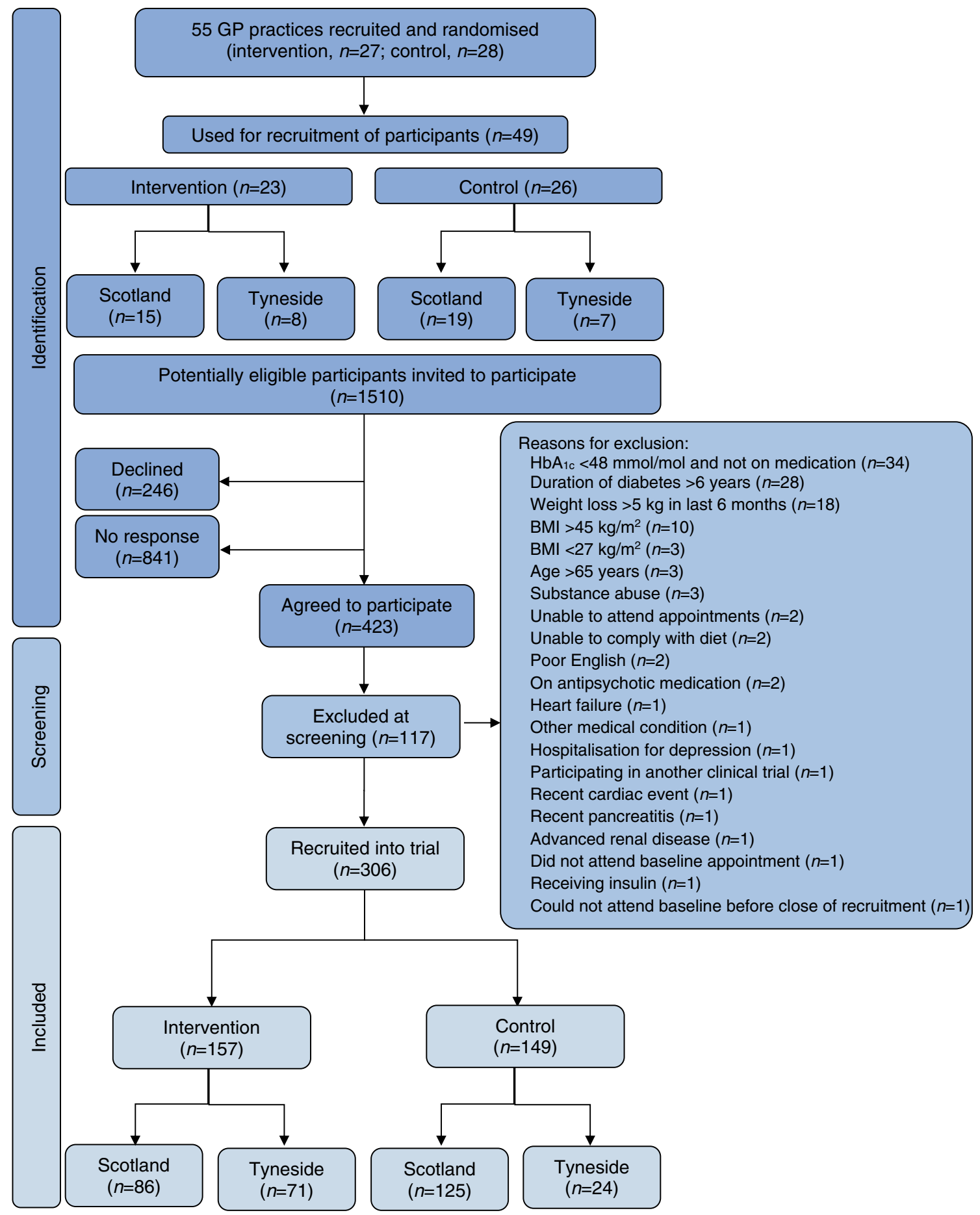

Fig. 1 Flowchart of recruitment 
there had been no such incentive for participants in control practices.

To ascertain whether the individuals volunteering and included were representative of the wider population with type 2 diabetes, defined by the inclusion and exclusion criteria, anonymised data on sex, height and weight, duration of diabetes and index of multiple deprivation were collated for all who were invited to participate.

Ethics approval for the Diabetes Remission Clinical Trial (DiRECT) was secured on 24 January 2014. NHS research and development approvals for participating Health Board areas in Scotland and Clinical Commissioning Groups in Tyneside were then secured. All subsequent protocol amendments have been similarly approved. Written informed consent was secured from all participants.

Statistical analyses Summary data are presented overall and by study area (Scotland, Tyneside). Continuous data are summarised by the mean and SD, and categorical data by frequencies and percentages. Study areas are compared using Wilcoxon-MannWhitney tests for continuous data and Fisher's exact tests for categorical data. Summary data are presented to enable comparison of the DiRECT study population with the wider Scottish population and with other studies of type 2 diabetes in the USA, Canada and Europe. Summary data are also reported for all individuals who were invited to take part in DiRECT and for whom anonymous baseline data could be obtained for comparison with the recruited study population. At this stage of analysis of baseline data, the codes have not been broken to reveal which participants are in which treatment arm and investigators remain blinded. Baseline data for the treatment arms will be analysed later, together with the primary outcome data.

\section{Results}

Primary care practice recruitment Of the 523 practices that received an invitation to participate in the study, 74 expressed interest in participating. Following meetings with the study team, 55 practices agreed to participate and were randomised (34 Scotland, 21 Tyneside). The main factor for nonparticipation was the potential time commitment required if allocated to the intervention arm. Four practices allocated to intervention and two to control were not required for recruitment. Therefore, 49 practices were asked to recruit participants (Fig. 1), with list sizes varying between 1400 and 30,000 individuals in both urban and rural areas.

Participant recruitment A total of 1510 potentially eligible people were invited to participate in the study (Fig. 1). Of these, $423(28 \%)$ accepted the invitation. The response was similar in the control and intervention arms (Table 1). Of those who wished to participate, $28 \%$ were excluded at screening. The most
Table 1 Recruitment response by treatment allocation and study area

\begin{tabular}{lccl}
\hline & Invited $(n)$ & Accepted $(n)$ & Response (\%) \\
\hline Intervention arm & & & \\
$\quad$ Scotland & 464 & 130 & 28 \\
Tyneside & 315 & 85 & 27 \\
$\quad$ Total & 779 & 215 & 28 \\
Control arm & & & \\
$\quad$ Scotland & 552 & 166 & 30 \\
$\quad$ Tyneside & 179 & 42 & 23 \\
$\quad$ Total & 731 & 208 & 28 \\
Total & 1510 & 423 & 28 \\
\hline
\end{tabular}

frequent reasons for exclusion were $\mathrm{HbA}_{1 \mathrm{c}}<48 \mathrm{mmol} / \mathrm{mol}$ (6.5\%) without glucose-lowering medication, duration of type 2 diabetes $>6$ years and recent weight loss $>5 \mathrm{~kg}$ (Fig. 1). From 48 practices, 306 participants gave consent and were recruited into the trial (no participants were recruited at one of the practices). After the baseline visit, one participant withdrew consent and seven participants were subsequently considered to have been randomised in error because their most recent $\mathrm{HbA}_{1 \mathrm{c}}$ was already in the non-diabetic range without drug treatment. These eight participants will not be included in the intention to treat (ITT) population for data analyses, and data are presented for the remaining 298 participants.

Baseline characteristics The baseline characteristics of the study population, detailed in Table 2, were similar for participants in Scotland and Tyneside. Mean age at recruitment was 54.4 years. Over half of the study population were male $(59 \%)$. Mean BMI was slightly higher in women $(35.3 \mathrm{~kg} /$ $\left.\mathrm{m}^{2}\right)$ than in men $\left(34.2 \mathrm{~kg} / \mathrm{m}^{2}\right)$. Mean $(\mathrm{SD}) \mathrm{HbA}_{1 \mathrm{c}}$ was 59.3 (12.7) $\mathrm{mmol} / \mathrm{mol}(7.6 \%$ [1.2\%]). Mean duration since diagnosis of type 2 diabetes was 3 years. Reflecting the ethnic composition of Scotland and Tyneside, 98\% of participants were white, with $0.7 \%$ Black African, $0.6 \%$ South Asian and $0.3 \%$ other. The majority of participants were prescribed glucoselowering medications $(n=226,76 \%)$, most as monotherapy ( $n=144,48 \%$ ), with metformin most commonly prescribed $(n=211,71 \%)$. About a quarter of participants were managed by diet alone, and $82(28 \%)$ were prescribed two or more glucose-lowering medications, including 63 (21\%) receiving sulfonylureas. Few participants (12\%) were current cigarette smokers and 50\% reported never having smoked. Only $26 \%$ of participants reported any current or recent weight management activities. Table 3 shows the documented medical history of participants at baseline. Twelve per cent of participants had existing retinopathy. More than half of the study population had a history of hypertension and $55 \%$ of participants were prescribed antihypertensive medication, with over $30 \%$ on two or more drugs. The overall prevalence of cardiovascular disease (CVD; myocardial infarction, angina, stroke, 
Table 2 Baseline characteristics by study area (ITT population)

\begin{tabular}{|c|c|c|c|c|}
\hline & All & Scotland & Tyneside & $p$ value \\
\hline$N$ & 298 & 197 & 101 & \\
\hline \multicolumn{5}{|l|}{ Sex, $n(\%)$} \\
\hline Male & $176(59.1)$ & $118(59.9)$ & $58(57.4)$ & \multirow[t]{2}{*}{0.710} \\
\hline Female & $122(40.9)$ & $79(40.1)$ & $43(42.6)$ & \\
\hline Age (years) & $54.4(7.6)$ & $55.2(7.2)$ & $52.8(8.1)$ & 0.014 \\
\hline \multicolumn{5}{|l|}{ Height (cm) } \\
\hline Men & $175.9(7.1)$ & $175.8(7.0)$ & $176.2(7.2)$ & 0.711 \\
\hline Women & $160.7(5.2)$ & $160.6(5.4)$ & $160.8(4.9)$ & 0.684 \\
\hline \multicolumn{5}{|l|}{ Weight (kg) } \\
\hline Men & $106.0(15.8)$ & $105.7(15.5)$ & $106.5(16.6)$ & 0.704 \\
\hline Women & $91.2(13.0)$ & $90.8(13.9)$ & $92.0(11.4)$ & 0.476 \\
\hline \multicolumn{5}{|l|}{ BMI $\left(\mathrm{kg} / \mathrm{m}^{2}\right)$} \\
\hline All & $34.6(4.4)$ & $34.6(4.4)$ & $34.8(4.4)$ & 0.656 \\
\hline Men & $34.2(4.2)$ & $34.2(4.2)$ & $34.2(4.4)$ & 0.929 \\
\hline Women & $35.3(4.6)$ & $35.1(4.8)$ & $35.6(4.4)$ & 0.548 \\
\hline \multicolumn{5}{|l|}{ Waist circumference $(\mathrm{cm})^{\mathrm{a}}$} \\
\hline Men & $110.0(7.1)$ & $110.7(6.2)$ & $108.6(8.7)$ & 0.148 \\
\hline Women & $101.6(8.6)$ & $101.7(9.3)$ & $101.4(7.1)$ & 0.831 \\
\hline \multicolumn{5}{|l|}{ Hip circumference $(\mathrm{cm})^{\mathrm{a}}$} \\
\hline Men & $108.6(5.6)$ & $109.4(5.6)$ & $107.1(5.3)$ & 0.066 \\
\hline Women & $111.6(8.0)$ & $111.9(8.4)$ & $111.0(7.1)$ & 0.804 \\
\hline Systolic blood pressure $(\mathrm{mm} / \mathrm{Hg})$ & $134.9(16.9)$ & $136.8(16.4)$ & $131.3(17.2)$ & 0.004 \\
\hline Diastolic blood pressure (mm/Hg) & $85.0(9.5)$ & $85.5(9.1)$ & $84.1(10.2)$ & 0.088 \\
\hline Duration of type 2 diabetes (years) & $3.0(1.7)$ & $3.0(1.7)$ & $2.9(1.7)$ & 0.710 \\
\hline \multicolumn{5}{|l|}{ Pre-trial $\mathrm{HbA}_{1 \mathrm{c}}$ (from GP records) } \\
\hline $\mathrm{mmol} / \mathrm{mol}$ & $61.6(13.9)$ & $61.4(14.2)$ & $62.0(13.4)$ & \multirow[t]{2}{*}{0.441} \\
\hline$\%$ & $7.79(1.27)$ & $7.76(1.30)$ & $7.83(1.23)$ & \\
\hline \multicolumn{5}{|l|}{ Trial measured $\mathrm{HbA}_{1 \mathrm{c}}$} \\
\hline $\mathrm{mmol} / \mathrm{mol}$ & $59.3(12.7)$ & $59.6(13.4)$ & $58.6(11.2)$ & \multirow[t]{2}{*}{0.880} \\
\hline$\%$ & $7.57(1.16)$ & $7.61(1.22)$ & $7.51(1.03)$ & \\
\hline \multicolumn{5}{|l|}{ Management of type 2 diabetes, $n(\%)$} \\
\hline Diet alone (no drugs) & $72(24.2)$ & $38(19.3)$ & $34(33.7)$ & \multirow[t]{3}{*}{0.018} \\
\hline 1 drug & $144(48.3)$ & $104(52.8)$ & $40(39.6)$ & \\
\hline$\geq 2$ drugs & $82(27.5)$ & $55(27.9)$ & $27(26.7)$ & \\
\hline \multicolumn{5}{|l|}{ Smoking status, $n(\%)$} \\
\hline Current & $35(11.7)$ & $21(10.7)$ & $14(13.9)$ & \multirow[t]{3}{*}{0.663} \\
\hline Former & $113(37.9)$ & $77(39.1)$ & $36(35.6)$ & \\
\hline Never & $150(50.3)$ & $99(50.3)$ & $51(50.5)$ & \\
\hline $\begin{array}{l}\text { Current/recent engagement in weight } \\
\text { management activities, } n(\%)\end{array}$ & $77(25.8)$ & $62(31.5)$ & $15(14.9)$ & 0.002 \\
\hline Reported weekly alcohol intake (units) & $5.5(9.6)$ & $5.5(10.3)$ & $5.7(8.0)$ & 0.040 \\
\hline
\end{tabular}

Data are mean (SD) unless otherwise stated

${ }^{\mathrm{a}}$ Waist and hip circumference was only measured when BMI was $<35 \mathrm{~kg} / \mathrm{m}^{2}$ transient ischaemic attack, heart failure, peripheral vascular disease, revascularisation, atrial fibrillation) was $12 \%$. Forty three per cent of participants were prescribed six or more drugs and $13 \%$ ten or more. The proportion being treated with antidepressant drugs was $23 \%$. Biochemical data at baseline (Table 4) were as expected among unselected individuals, predominantly with obesity and within 6 years of type 2 diabetes diagnosis.

\section{Discussion}

DiRECT has a realistic or 'pragmatic' design to provide information about the management of type 2 diabetes in a routine primary care setting in a largely unselected population in order to maximise transferability of results. Certain inclusion criteria were applied for recruitment into DiRECT for various 
Table 3 Medical history documented at baseline by study area (ITT population)

\begin{tabular}{|c|c|c|c|c|}
\hline & All & Scotland & Tyneside & $p$ value \\
\hline$N$ & 298 & 197 & 101 & \\
\hline Diabetic retinopathy & $35(11.7)$ & $20(10.2)$ & $15(14.9)$ & 0.256 \\
\hline Hypertension $^{\mathrm{a}}$ & $169(56.7)$ & $113(57.4)$ & $56(55.4)$ & 0.805 \\
\hline Current antihypertensive medication & $163(54.7)$ & $113(57.4)$ & $50(49.5)$ & 0.220 \\
\hline $\begin{array}{l}1 \text { drug } \\
\geq 2 \text { drugs }\end{array}$ & $\begin{array}{l}69(23.2) \\
94(31.5)\end{array}$ & $\begin{array}{l}45(22.8) \\
68(34.5)\end{array}$ & $\begin{array}{l}24(23.8) \\
26(25.7)\end{array}$ & 0.279 \\
\hline Current antidepressant medication & $68(22.8)$ & $44(22.3)$ & $24(23.8)$ & 0.773 \\
\hline \multicolumn{5}{|l|}{ Total number of prescribed medications } \\
\hline $\begin{array}{l}\text { None } \\
1-2\end{array}$ & $\begin{array}{l}6(2.0) \\
47(15.8)\end{array}$ & $\begin{array}{l}3(1.5) \\
30(15.2)\end{array}$ & $\begin{array}{l}3(3.0) \\
17(16.8)\end{array}$ & 0.280 \\
\hline $3-5$ & $116(38.9)$ & $73(37.1)$ & $43(42.6)$ & \\
\hline $6-9$ & 89 (29.9) & $59(29.9)$ & $30(29.7)$ & \\
\hline$\geq 10$ & $40(13.4)$ & $32(16.2)$ & $8(7.9)$ & \\
\hline \multicolumn{5}{|l|}{ Clinical CVD } \\
\hline Myocardial infarction & $8(2.7)$ & $7(3.6)$ & $1(1.0)$ & 0.273 \\
\hline Angina & $8(2.7)$ & $6(3.0)$ & $2(2.0)$ & 0.721 \\
\hline Stroke & $7(2.3)$ & $5(2.5)$ & $2(2.0)$ & 1.000 \\
\hline Transient ischaemic attack & $7(2.3)$ & $7(3.6)$ & $0(0.0)$ & 0.100 \\
\hline Heart failure & $2(0.7)$ & $2(1.0)$ & $0(0.0)$ & 0.550 \\
\hline Peripheral vascular disease & $7(2.3)$ & $5(2.5)$ & $2(2.0)$ & 1.000 \\
\hline Previous revascularisation & $4(1.3)$ & $4(2.0)$ & $0(0.0)$ & 0.304 \\
\hline Atrial fibrillation & $8(2.7)$ & $7(3.6)$ & $1(1.0)$ & 0.273 \\
\hline Any CVD & $37(12.4)$ & $30(15.2)$ & $7(6.9)$ & 0.042 \\
\hline Hyperlipidaemia $^{\mathrm{b}}$ & $67(22.5)$ & $48(24.4)$ & $19(18.8)$ & 0.307 \\
\hline $\mathrm{eGFR}<60 \mathrm{ml} \mathrm{min}{ }^{-1} 1.73 \mathrm{~m}^{-2 \mathrm{c}}$ & $9(3.1)$ & $7(3.6)$ & $2(2.1)$ & 0.723 \\
\hline Arthritis & $58(19.5)$ & $39(19.8)$ & $19(18.8)$ & 0.878 \\
\hline Osteoporosis & $2(0.7)$ & $2(1.0)$ & $0(0.0)$ & 0.550 \\
\hline Epilepsy & $2(0.7)$ & $1(0.5)$ & $1(1.0)$ & 1.000 \\
\hline Other & $237(79.5)$ & $147(74.6)$ & $90(89.1)$ & 0.004 \\
\hline
\end{tabular}

Data presented as $n(\%)$

${ }^{\text {a }}$ Hypertension is defined as in the National Institute for Health and Care Excellence (NICE) guidelines for type 2 diabetes [25]

${ }^{\mathrm{b}}$ Hyperlipidaemia is defined as in the NICE guidelines for cardiovascular disease [26]

${ }^{\mathrm{c}}$ For eGFR, $n=196$ for Scotland, $n=95$ for Tyneside and $n=291$ for Au (missing data are owing to missing creatinine values at baseline) practical reasons. An upper BMI limit of $45 \mathrm{~kg} / \mathrm{m}^{2}$ was set because the protocol required a subsample to undergo abdominal magnetic resonance imaging. An upper age limit of 65 years was fixed to avoid the greater mortality rates associated with older people in a study planned to continue for 2 years and to optimise attendance at study visits given the greater mobility problems often faced by older people with type 2 diabetes. Furthermore, individuals were excluded if they had suffered myocardial infarction or stroke within the 6 months prior to recruitment because of engagement in other programmes.

Volunteers for clinical research are usually relatively more health conscious and there can be a risk of attracting unrepresentative 'concerned but healthy' individuals. The DiRECT cohort included, at baseline, $12 \%$ with existing retinopathy,
$12 \%$ with one or more known manifestations of heart disease and $13 \%$ with microalbuminuria indicating nephropathy. Impaired renal function (eGFR <60) was present in 3\%. In addition to their diabetes, as may be expected, a high proportion (57\%) had known hypertension and $31 \%$ were prescribed two or more antihypertensive medications. Polypharmacy was common, with $43 \%$ being prescribed six or more different drugs daily and $13 \%$ prescribed ten or more drugs. Almost a quarter of the entire cohort was prescribed antidepressant medications, some of which are associated with weight gain [11]. Thus, the DiRECT cohort does not appear to include an excess of 'worried well' or unusually healthy people with type 2 diabetes.

DiRECT recruited a substantial proportion of men. This is encouraging as this is in line with the higher prevalence of 
Table 4 Baseline biochemistry by study area (ITT population)

\begin{tabular}{lcccr}
\hline & All & Scotland & Tyneside & $p$ value \\
\hline$n$ (missing) & $291(7)$ & $196(1)$ & $95(6)$ & \\
Total cholesterol (mmol/l) & $4.32(1.19)$ & $4.38(1.21)$ & $4.19(1.13)$ & 0.239 \\
HDL-cholesterol (mmol/l) & $1.12(0.28)$ & $1.14(0.28)$ & $1.08(0.29)$ & 0.043 \\
Triacylglycerol (mmol/l) & $2.00(1.17)$ & $2.10(1.27)$ & $1.80(0.89)$ & 0.034 \\
ALT (U/l) & $34.1(19.4)$ & $34.8(20.1)$ & $32.7(17.8)$ & 0.450 \\
AST (U/l) & $24.2(12.8)$ & $24.8(13.1)$ & $22.8(12.2)$ & 0.136 \\
GGT (U/l) & $52.4(56.8)$ & $54.5(62.8)$ & $48.0(41.7)$ & 0.567 \\
Creatinine $(\mu \mathrm{mol} / \mathrm{l})$ & $68.3(15.1)$ & $70.7(15.1)$ & $63.3(13.9)$ & $<0.001$ \\
eGFR (ml min $\left.{ }^{-1} 1.73 \mathrm{~m}^{-2}\right)$ & $98.6(24.7)$ & $94.3(22.0)$ & $107.5(27.4)$ & $<0.001$ \\
$n$ (missing) & $292(6)$ & $196(1)$ & $96(5)$ & \\
Glucose (mmol/l) & $9.01(2.94)$ & $9.30(2.94)$ & $8.43(2.85)$ & 0.010 \\
Insulin $\left(\mathrm{mU} / \mathrm{ml}^{2}\right)$ & $23.50(14.35)$ & $25.34(14.58)$ & $19.67(13.13)$ & $<0.001$ \\
$n$ (missing) & $292(6)$ & $197(0)$ & $95(6)$ & \\
ACR (mg/mmol) & $2.16(6.89)$ & $1.71(6.73)$ & $3.08(7.16)$ & 0.414 \\
Microalbuminuria, $n(\%)^{\mathrm{b}}$ & $39(13.4)$ & $18(9.1)$ & $21(22.1)$ & 0.003 \\
\hline
\end{tabular}

Data are mean (SD) unless otherwise stated

${ }^{\text {a }}$ Values $<0.5$ imputed as 0.25

${ }^{\mathrm{b}}$ Defined as ACR $\geq 3.5$ (female) or ACR $\geq 2.5$ (male) type 2 diabetes in men [12]. Comparison of the study population with available data for those invited to participate in DiRECT shows an almost identical proportion of men and women accepting the invitation to participate (Table 5). Typically, men are less likely to engage in weight management programmes [13-15]. However, the potential for health improvement is a motivator for weight loss in men $[16,17]$, so

Table 5 Comparison of invited participants with the DiRECT study population

\begin{tabular}{lll}
\hline & Invited population & DiRECT participants \\
\hline$n$ & $1155^{\mathrm{a}}$ & 298 \\
Sex, $n(\%)$ & & \\
$\quad$ Male & $699(61.3)$ & $176(59.1)$ \\
$\quad$ Female & $442(38.7)$ & $122(40.9)$ \\
Year of birth & $1961(8)$ & $1961(8)$ \\
BMI (kg/m $\left.{ }^{2}\right)$ & $33.5(6.9)$ & $34.6(4.4)$ \\
Duration of type 2 & $3.5(3.2)$ & $3.0(1.7)$ \\
$\quad$ diabetes (years) & & \\
Index of Multiple Deprivation quintile, $n(\%)$ & \\
$\quad$ Q1 - Most deprived & $257(22.8)$ & $63(21.4)$ \\
Q2 & $185(16.4)$ & $52(17.6)$ \\
Q3 & $226(20.0)$ & $64(21.7)$ \\
$\quad$ Q4 & $238(21.1)$ & $67(22.7)$ \\
Q5 - Least deprived & $222(19.7)$ & $49(16.6)$ \\
\hline
\end{tabular}

Data are mean (SD) unless otherwise stated

${ }^{a}$ Data available for 1141 invited participants. Missing data for each item: sex, $n=14$ (invited); year of birth, $n=10$ (invited); BMI, $n=22$ (invited); duration of diabetes, $n=603$ (invited); deprivation, $n=30$ (invited $=27$; $\operatorname{DiRECT}=3$ ) a potential remission of diabetes or improvement in diabetes status may have encouraged men to participate in DiRECT. Whether a total diet replacement approach might be more attractive to men than to women, and other motivational issues, will be explored in interviews with participants and with healthcare professionals involved in DiRECT.

Recruitment of participants to any study is challenging. Poor recruitment can result in inadequate study numbers and underpowered trials [18]. DiRECT was based in primary care in order to provide realistic results transferable to routine practice. Of the invited practices, $14 \%$ accepted, reflecting the relatively small proportion of general practices in the UK that are research-active. Many of those are at capacity for taking on new research projects, being busy NHS general practices with limited availability of doctor and nurse time for research. There was great interest in DiRECT, but many of the research-active practices had already committed to research studies on other topics. Moreover, practice participation required practices to be able to provide six to ten individuals who would meet the inclusion criteria; this excluded some smaller practices. Recruitment to DiRECT was monitored closely and recruitment methods were refined to include strategies known to maximise recruitment (e.g. reminder letter, telephone contact and incentive) [18]. It is remarkable that $28 \%$ of all invited to participate expressed interest in doing so, and most of those who initially volunteered participated $(72 \%)$. This far exceeds many other trials that recruited using similar methods [19] and those that recruited by other means $[20,21]$. The study recruited more than the numbers required to satisfy the a priori power calculation (140 per group) within the 2 years allocated for recruitment, and recruitment proved 
difficult to halt resulting in modest over-recruitment. The ready acceptance of a low-energy liquid diet has been observed in all of our previous studies [1, 3, 9].

Triacylglycerol, insulin, fasting glucose and eGFR were less good in Scottish participants, in keeping with the slightly greater age and higher waist circumferences. None of these differences could be considered clinically important. Average alanine aminotransferase (ALT) and $\gamma$-glutamyl transferase (GGT) levels were higher than the normal reference range. There were a small number with ALT and GGT raised more so than aspartate aminotransferase (AST). This will be partly the result of fatty liver, which is common in this participant group, but more substantial elevations in GGT without comparable ALT elevations suggest that some participants may have had undisclosed alcohol excess. Of the 12 participants with GGT >150 at baseline, only one reported very high alcohol consumption (72 units per week). In most instances transaminases were also elevated but there were no other features in the baseline data which might account for abnormal liver function tests. There were minor excesses of high albumin/creatinine ratio (ACR) in Tyneside, the reasons for which were not clear although the numbers are small and do not allow determination of whether such minor albuminuria was sustained or transient.

The participants can be considered representative of the general population of people with short duration type 2 diabetes, and their characteristics were similar to those of other large studies of type 2 diabetes (Table 6). Comparison with
LookAHEAD [20] showed similar mean $\mathrm{HbA}_{1 \mathrm{c}}(55 \mathrm{mmol} /$ mol [7.2\%]) and BMI $\left(35.9 \mathrm{~kg} / \mathrm{m}^{2}\right)$, low prevalence of CVD $(14.1 \%)$ and a high proportion of participants never having smoked $(50.2 \%)$, although the proportion of male participants was lower (40\%) and the population slightly older. The prevalence of CVD was higher in the ACCORD [21] and PROactive [22] studies, but this was a specific inclusion criterion for both those studies. As in DiRECT, both of these studies had high proportions of male participants and a similar mean BMI. Median $\mathrm{HbA}_{1 \mathrm{c}}$ was higher in ACCORD and PROactive (64 mmol/mol [8\%]). Importantly, duration of diabetes was longer in all three of these previous studies, while it was specifically limited to $<6$ years in the present study. This duration was selected in view of the demonstration of duration of type 2 diabetes being a critical factor in achieving remission [3]. Our study population was, however, very representative of all people with type 2 diabetes in Scotland, in whom the mean duration of diabetes was 9 years [23]. A greater number of medications were prescribed to the Scottish than to the Tyneside participants, perhaps reflecting variations in the application of the different clinical guidelines in Scotland [24] and England [25].

A remarkably high proportion of eligible people with type 2 diabetes volunteered to enter DiRECT, including more men than is usual for weight management trials. The participants recruited were broadly representative of the unselected general population of people with up to 6 years' duration of type 2 diabetes, and the subset in Tyneside were similar to the Scottish subset. The recruitment rates into the intervention

Table 6 Comparison of DiRECT participant characteristics with those of the wider population of people with type 2 diabetes in Scotland and with those recruited into other type 2 diabetes studies

\begin{tabular}{|c|c|c|c|c|c|}
\hline & DiRECT & $\begin{array}{l}\text { Scottish population of people } \\
\text { with type } 2 \text { diabetes } \\
(2015 / 2016)[23]\end{array}$ & $\begin{array}{l}\text { LookAHEAD } \\
{[20] \text { (USA) }}\end{array}$ & $\begin{array}{l}\text { ACCORD [21] } \\
\text { (USA/Canada) }\end{array}$ & $\begin{array}{l}\text { PROactive } \\
\text { [22] (Europe) }\end{array}$ \\
\hline$N$ & 298 & 263,843 & 5145 & 10,251 & 5238 \\
\hline \multicolumn{6}{|l|}{$\operatorname{Sex}(\%)$} \\
\hline Male & 59.1 & 57.5 & 40.6 & 61.3 & 66.5 \\
\hline Female & 40.9 & 42.5 & 59.4 & 38.7 & 33.5 \\
\hline Mean age (years) & 54.4 & 66.7 & 59.0 & 62.2 & 61.8 \\
\hline Mean BMI $\left(\mathrm{kg} / \mathrm{m}^{2}\right)$ & 34.6 & 32.0 & 35.9 & 32.2 & 31.0 \\
\hline Duration of type 2 diabetes (years) & 3.0 & 9.3 & 7.0 & 10 & 8 \\
\hline \multicolumn{6}{|l|}{ Mean $\mathrm{HbA}_{1 \mathrm{c}}$} \\
\hline $\mathrm{mmol} / \mathrm{mol}$ & 59.3 & 59.1 & 56.0 & 67.0 & 63.0 \\
\hline$\%$ & 7.6 & 7.6 & 7.3 & 8.3 & 7.9 \\
\hline History of hypertension (\%) & 56.7 & $\mathrm{n} / \mathrm{a}$ & 80.3 & $\mathrm{n} / \mathrm{a}$ & 75.6 \\
\hline Antihypertensive medication (\%) & 54.7 & $\mathrm{n} / \mathrm{a}$ & 68.4 & 85.5 & $\mathrm{n} / \mathrm{a}$ \\
\hline \multicolumn{6}{|l|}{ Smoking status (\%) } \\
\hline Current & 11.7 & $\mathrm{n} / \mathrm{a}$ & 4.4 & 14.0 & 13.5 \\
\hline Former & 37.9 & & 45.4 & 44.0 & 45 \\
\hline Never & 50.3 & & 50.2 & 42.0 & $\mathrm{n} / \mathrm{a}$ \\
\hline History of CVD (\%) & 12.4 & $\mathrm{n} / \mathrm{a}$ & 14.1 & 35.0 & $\mathrm{n} / \mathrm{a}$ \\
\hline
\end{tabular}


and control arms were almost identical. The results of the weight management intervention in DiRECT are thus likely to be widely applicable to people with type 2 diabetes.

Acknowledgements We thank NHS PCRN and NECS for their support and valuable input to recruitment. We thank E. Butler, S. J. Duffus, P. Stewart and J. Cooney, University of Glasgow, for technical assistance, and research nurse H. Pilkington, Newcastle upon Tyne Hospitals NHS Foundation Trust. We are enormously grateful to the GP practices, healthcare professionals and volunteers for their participation.

Data availability The datasets generated and/or analysed during the current study are available from the corresponding author on reasonable request.

Funding The DiRECT study was funded as a Strategic Research Initiative by Diabetes UK (award number 13/0004691). The formula diet was donated by Cambridge Weight Plan. Neither organisation had any input into the study design, data analysis/interpretation, writing or editing, or the decision to publish. AA is funded by the National Institute for Health Research (NIHR) as an NIHR Research Professor.

Duality of interest $\mathrm{ML}$ and NB have received funding from Cambridge Weight Plan for conference attendance and for other departmental research. Prior to study commencement, NB, HR and LMcC were shareholders and employees of Counterweight Ltd. HR and NB remain employees and shareholders of Counterweight Ltd. ML has provided consultancy to Counterweight Ltd.

Contribution statement $\mathrm{ML}$ and RT conceived the study and are the principal investigators. All authors contributed to the design of the study. $\mathrm{WL}$ is the trial coordinator and coordinated recruitment and acquisition of study data. YMcI coordinated the recruitment of GP practices. NB, GT, $\mathrm{LMcC}$ and $\mathrm{AB}$ recruited participants and contributed to the acquisition of data. SK and IF managed the study data and $\mathrm{AMcC}$ performed the statistical analyses. PW and NS conducted the biochemical analyses. CP, SZ, $\mathrm{KH}, \mathrm{JM}$ and AAM contributed to the acquisition, analyses and interpretation of mechanistic study data. HR, a grant holder on the study, provided expertise on the Counterweight-Plus programme delivery. FS, AR, LR and AA contributed to the acquisition, analyses and interpretation of qualitative data. ML, WL and RT drafted the manuscript. All authors critically reviewed and revised the manuscript, and have read and approved the final version. All authors agree to be accountable for all aspects of the work in respect of accuracy and integrity. Overall, ML and RT are the guarantors of this work, had full access to all study data, and take responsibility for the integrity of the data and the accuracy of the data analyses.

Open Access This article is distributed under the terms of the Creative Commons Attribution 4.0 International License (http:// creativecommons.org/licenses/by/4.0/), which permits unrestricted use, distribution, and reproduction in any medium, provided you give appropriate credit to the original author(s) and the source, provide a link to the Creative Commons license, and indicate if changes were made.

\section{References}

1. Lim EL, Hollingsworth KG, Aribisala BS, Chen MJ, Mathers JC, Taylor R (2011) Reversal of type 2 diabetes: normalisation of beta cell function in association with decreased pancreas and liver triacylglycerol. Diabetologia 54:2506-2514
2. Steven S, Lim E, Taylor R (2013) Population response to information on reversibility of type 2 diabetes. Diabet Med 30:e135-e138

3. Steven S, Hollingsworth KG, Al-Mrabeh A et al (2016) Very low calorie diet and 6 months of weight stability in type 2 diabetes: pathophysiological changes in responders and nonresponders. Diabetes Care 39:158-165

4. Guidone C, Manco M, Valera-Mora E et al (2006) Mechanisms of recovery from type 2 diabetes after malabsorptive bariatric surgery. Diabetes 55:2025-2031

5. Steven S, Carey PE, Small PK, Taylor R (2015) Reversal of type 2 diabetes after bariatric surgery is determined by the degree of achieved weight loss in both short- and long-duration diabetes. Diabet Med 32:47-53

6. Dixon J, Chuang LM, Chong K et al (2013) Predicting the glycemic response to gastric bypass surgery in patients with type 2 diabetes. Diabetes Care 36:20-26

7. McCombie L, Leslie W, Taylor R, Kennon B, Sattat N, Lean MEJ (2017) Beating diabetes with IT coding of 'diabetes in remission': an emerging new target for patients. BMJ 358:j4030

8. Gregg EW, Chen H, Wagenknecht LE et al (2012) Association of an intensive lifestyle intervention with remission of type 2 diabetes. JAMA 308:2489-2496

9. Lean M, Brosnahan N, McLoone P et al (2013) Feasibility and indicative results from a 12-month low-energy liquid diet treatment and maintenance programme for severe obesity. Br J Gen Pract 63: e115-e124

10. Leslie WS, Ford I, Sattar N et al (2016) The Diabetes Remission Clinical Trial (DiRECT): protocol for a cluster randomised trial. BMC Fam Pract 17:20

11. Leslie WS, Hankey CR, Lean MEJ (2007) Weight gain as an adverse effect of some commonly prescribed drugs: as systematic review. QJM 100:395-404

12. Logue J, Walker JJ, Colhoun HM et al (2011) Do men develop type 2 diabetes at lower body mass indices than women? Diabetologia 54:3003-3006

13. Ahern AL, Aveyard P, Boyland EJ, Halford JCG, Jebb SA (2016) Inequalities in the uptake of weight management interventions in a pragmatic trial: an observational study in primary care. Br J Gen Pract 66:e258-e263

14. Booth HP, Prevost AT, Gulliford MC (2015) Access to weight reduction interventions for overweight and obese patients in UK primary care: population-based cohort study. BMJ Open 5:e006642

15. Robertson C, Avenell A, Boachie C et al (2015) Should weight loss and maintenance programmes be designed differently for men? A systematic review of long-term randomised controlled trials presenting data for men and women: The ROMEO project. Obes Res Clin Pract 10:70-84

16. Hankey CR, Leslie WS, Lean MEJ (2002) Why lose weight? Reasons for seeking weight loss by overweight but otherwise healthy men. Int J Obes 26:880-882

17. De Souza P, Ciclitira E (2005) Men and dieting: a qualitative analysis. J Health Psychol 10:793-804

18. Treweek S, Lockhart, Pitkethly M et al (2013) Methods to improve recruitment to randomised controlled trials: Cochrane systematic review and meta-analysis. BMJ Open 3:e002360

19. Khunti K, Taub N, Tringham J et al (2010) Screening for the metabolic syndrome using simple anthropometric measurements in South Asian and white Europeans: a population-based screening study. The Leicester Ethnic Atherosclerosis and Diabetes Risk (LEADER) Study. Prim Care Diabetes 4:25-32

20. The LookAHEAD Research Group (2006) Baseline characteristics of the randomized cohort from the LookAHEAD (Action for Health in Diabetes) research study. Diab Vasc Dis Res 3:202-215

21. Action to Control Cardiovascular Risk in Diabetes Study Group (2008) Effects of intensive glucose lowering in type 2 diabetes. N Engl J Med 358:2545-2559 
22. Dormandy JA, Charbonnel B, Eckland DJA et al (2005) Secondary prevention of macrovascular events in patients with type 2 diabetes in the PROactive Study (PROspective pioglitazone Clinical Trial in macroVascular Events): a randomised controlled trial. Lancet 366: 1279-1289

23. Brierley L, McGurnaghan S, Blackbourn L, McKeigue PM, Colhoun HM (2017) The changing landscape of diabetes drug therapy in a national population of people with type 2 diabetes. Diabetologia 60(Suppl 1):A849 (Abstract)
24. Scottish Intercollegiate Guideline Network (2010) Management of diabetes: a national clinical guideline. http://www.sign.ac.uk/assets/ sign116.pdf accessed 6 Nov 2017

25. National Institute for Health and Care Excellence (2015) Type 2 diabetes in adults: management https://www.nice.org.uk/guidance/ ng28 accessed 6 Nov 2017

26. National Institute for Health and Care Excellence (2014) Cardiovascular disease: risk assessment and reduction, including lipid modification https://www.nice.org.uk/guidance/cg181 accessed 6 Nov 2017 